\title{
The impact of diffuse large B-cell lymphoma-derived exosomes on macrophage polarisation and cytokine release
}

Huayu Ling ${ }^{1}$, Zhong Yang ${ }^{2}$, Yu Sun ${ }^{1}$, Yuanning $\mathrm{He}^{1}$, Songguang $\mathrm{Ju}^{3}$, Jun $\mathrm{Li}^{1}$, Jinxiang $\mathrm{Fu}^{1}$

\author{
${ }^{1}$ Haematology Department, The Second Affiliated Hospital of Soochow University, \\ Suzhou, China \\ ${ }^{2}$ Nuclear Medicine Department, BOE Hospital, HeFei, China \\ ${ }^{3}$ Institute of Biotechnology, Soochow University, Suzhou, China
}

Submitted: 9 February 2020

Accepted: 25 June 2020

Arch Med Sci

DOI: https://doi.org/10.5114/aoms.2020.97355

Copyright $\odot 2020$ Termedia \& Banach

\begin{abstract}
Introduction: The pro-tumoural role of $M 2$ macrophages has been described in various cancers and is associated with poor clinical outcomes. Exosomes derived from cancers can shuttle information among tumour cells, immune cells, and the microenvironment, leading to microenvironment remodelling. The mechanism involved in exosomes influencing macrophages polarisation is still unclear.

Material and methods: We established a macrophage model derived from THP-1 monocytes. To mimic the in vivo microenvironment, we generated an indirect co-culture system of macrophages with diffuse large B-cell lymphoma (DLBCL) cell lines, OCI-LY1 and OCI-LY3 cells. In addition, pre-treated macrophages with exosomes derived from OCI-LY1/3 lymphoma cells were used in our study. The changes in macrophage polarisation stage, surface receptor expression, and cytokine expression at the mRNA level were analysed. Moreover, the effect of macrophages on drug-induced lymphoma cell apoptosis in the indirect co-culture system was assessed. Furthermore, protein analysis of NF- $\kappa \mathrm{B}$ and p-p65 in macrophages exposed to exosomes was performed by western blot.

Results: DLBCL-derived exosomes had different effects on macrophages at different differentiation stages; these macrophages had different effects on drug-induced apoptosis of lymphoma cells in indirect co-culture. Moreover, NF- $\mathrm{KB}$ signalling molecules were involved in this process.

Conclusions: The results indicate that DLBCL-derived exosomes can lead to more $\mathrm{M} 2$ phenotypes, promote tumour growth, and play a substantial role in tumour progression, invasion, and drug-resistance.
\end{abstract}

Key words: macrophages, tumour microenvironment, diffuse large B-cell lymphoma, exosome, nuclear factor- $\kappa$ B.

\section{Introduction}

Diffuse large B-cell lymphoma (DLBCL) is the most common subtype of lymphoma, occupying $30-40 \%$ of preliminary diagnosed non-Hodgkin's lymphomas; approximately $30 \%$ of patients with late stage DLBCL remain intractable and relapse [1]. The tumour microenvironment (TME) plays an important role in the proliferation, angiogenesis, migration, and metastasis of tumours by promoting the secretion of growth factors, an-

\author{
Corresponding author: \\ Jinxiang Fu PhD \\ Haematology Department \\ The Second Affiliated \\ Hospital of \\ Soochow University \\ Suzhou 215004, China \\ E-mail: fujinxiang@suda. \\ edu.cn
}


giogenic factors, and adhesion molecules [2]. Evidence shows that DLBCL is not purely the result of autonomous cell growth but also relies on the survival and proliferation signals from the microenvironment [3].

Macrophages are derived from two different pools of circulating inflammatory or resident monocytes that are recruited to tissues in response to inflammation or infection, and they also maintain homeostasis by replacing apoptotic resident macrophages [4, 5]. The microenvironment has a great impact on macrophage heterogeneity and plasticity in anti- and pro-inflammatory immune processes [6]. During the early stage of tumour development, monocytes recruited to the tumour site encounter proinflammatory signals that contribute to their differentiation into $M 1$ macrophages. In later stages of neoplastic transformation, signals from the tumour and neighbouring stromal cells promote changes toward an M2 macrophage phenotype, supporting further tumour progression [7]. Several studies have indicated that tumour-associated macrophages (TAMs) are associated with the poor clinical outcome of patients with DLBCL, even under rituximab treatment [8-10]. However, the mechanism of communication between lymphoma cells and macrophages in the TME is not fully understood.

Current knowledge of exosomes shows that they play an important role in the development and progression of cancer, modulate intercellular communication within the TME by transferring proteins, lipids, and genetic content [11, 12], and promote the $\mathrm{M} 2$ phenotypic functional polarisation of TAMs [13]. Previous studies have shown that cancer-derived exosomes can acclimate immune cells to a pro-tumour phenotype, and significantly contribute to tumour progression, including the promotion of tumourigenesis, invasion, angiogenesis, dissemination, and formation of pre-metastatic niches [14]. Importantly, the recruitment and migration of immune cells into TME is governed by dynamic signalling, and exosomes are a key component of these interactions [15]. However, the knowledge of tumour exosomes affecting macrophages in different differentiated stages in the TME remains unclear.

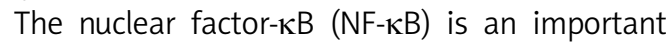
transcription factor of genes. Many cytokines are regulated by $\mathrm{NF}-\mathrm{\kappa B}$ in the early stages of the immune response and at various stages of the inflammatory response [16]. NF- $\mathrm{KB}$ plays a key role in inflammatory injury, tumour formation, angiogenesis, metastasis, and cell apoptosis [17]. We hypothesise that tumour exosomes affect the phenotype of macrophages and determine whether NF-KB signalling is involved in this process. Hence, in this study, we investigated the activation of NF-KB signalling in macrophages treated with lymphoma exosomes. This study will provide novel insight into the underlying mechanisms of communication between cancer exosomes and macrophages in the TME.

\section{Material and methods}

\section{Cell culture}

Human DLBCL cell lines, non-germinal centre B-cell-like (non-GCB) subtype OCI-LY3, and GCB subtype OCI-LY1 and SU-DHL8 cells were grown in Iscove's Modified Dulbecco's Medium (Life Technologies Corporation, NY, USA) supplemented with $10 \%$ foetal calf serum (FCS, Hyclone, Loan, UT, USA). The human monocytic THP-1 cells were maintained in RPMI 1640 medium supplemented with 10\% FCS. THP-1 monocytes were differentiated into inactivated $\mathrm{MO}$ macrophages for $24 \mathrm{~h}$ by incubation with $50 \mathrm{ng} / \mathrm{ml}$ phorbol 12-myristate 13-acetate (SigmaAldrich, St. Louis, MO, USA) followed by another 24-h incubation in complete RPMI medium.

\section{Exosome purification and identification}

Exosomes were purified from supernatants of 48-h-cultured lymphoma cells by ultracentrifugation combined with use of an exosome extraction kit (Thermo Fisher Scientific, MA, USA). Briefly, dead cells and debris were removed by centrifugation and use of an exosome extraction kit according to the manufacturer's instruction. A limited number of samples were selected to verify the morphology of vesicles using transmission electron microscopy (TEM). To further validate the purity of exosome preparations, after quantifying the amount of protein using the BCA protein assay, western blotting was performed to measure the levels of CD9 and CD63.

\section{Macrophage polarisation and indirect co-culture with DLBCL cells}

After THP-1 monocytes were differentiated into MO macrophages, MO macrophages were polarised into $\mathrm{M} 2$ macrophages by incubation with $20 \mathrm{ng} / \mathrm{ml}$ of IL-4 and IL-13. In the co-culture assay, lymphoma cells in RPMI 1640 were plated into transwell chambers (24 mm, $0.4 \mu \mathrm{m}$ microporous; Corning Inc., NY, USA), and THP-1 monocytes $\left(1 \times 10^{6}\right)$ were differentiated and polarised in six-well plates under the chambers. Macrophages treated or untreated with DLBCL exosomes were co-cultured with DLBCL cells in RPMI medium for $24 \mathrm{~h}$ before incubation with epirubicin for another $24 \mathrm{~h}$.

\section{DiO-labelling of exosomes and tracking}

For the fluorescence labelling of exosomes, exosomes were exposed to $5 \mathrm{mg} / \mathrm{l}$ solution of $\mathrm{DiO}$ (Thermo Fisher Scientific, MA, USA) membrane dye, 
according to the manufacturer's instructions, incubated in the dark at $37^{\circ} \mathrm{C}$ for 30 min and re-purified by ultracentrifugation and an exosome extraction kit. Briefly, macrophages were incubated for $4 \mathrm{~h}$ with $10 \mu \mathrm{g} / \mathrm{ml}$ DiO-labelled exosomes at $37^{\circ} \mathrm{C}$. After incubation, the cells were washed with PBS. Finally, images were obtained using a laser scanning confocal microscopy (Carl Zeiss AG, Jena, GER).

\section{Co-culture of THP-1-derived macrophages and exosomes}

Isolated exosomes were quantified using the BCA protein assay and administered to macrophages in equal amounts. After THP-1 monocyte differentiation and polarisation, these cells were treated with DLBCL-derived exosomes in equal amounts for $48 \mathrm{~h}$.

\section{Flow cytometric analysis of drug-induced apoptosis in DLBCL cells indirectly co-cultured with macrophages}

For analysis of apoptosis of DLBCL cells under indirect co-culture conditions, OCI-LY1 or $\mathrm{OCl}-\mathrm{LY} 3$ cells were co-cultured with $\mathrm{MO}$ and $\mathrm{M} 2$ macrophages treated or untreated with $\mathrm{DLBCL}$ exosomes. After co-culture, DLBCL cells were harvested and stained using an Annexin V-FITC/PI apoptosis Detection Kit and prepared according to the manufacturer's instructions. Results were analysed by flow cytometry (FCM, Beckman Coulter, CytoFLEX, CA, USA).

\section{Extraction of protein and western blotting}

Exosome and cell precipitation were lysed in RIPA lysis buffer containing a protease inhibitor cocktail (Roche, Basel, Switzerland) and a phosphatase inhibitor cocktail (Cell Signaling Technology, USA), and the total cytoplasmic protein was harvested. In brief, the precipitant was collected and lysed with RIPA buffer for 30 min on ice, centrifuged at $14,000 \times \mathrm{g}$ for $15 \mathrm{~min}$, the supernatant was transferred to a new Ep tube, and the protein was obtained. The protein concentrations were normalised using a BCA protein assay. Protein extracts were subjected to SDS-PAGE, transferred onto polyvinylidene fluoride membranes, and blocked with $1 \times$ TBS buffer containing $0.1 \%$ Tween 20 and $5 \%(\mathrm{w} / \mathrm{v})$ skimmed milk powder or $5 \%$ BSA. Then, the protein extracts were probed with primary antibodies, followed by an anti-rabbit or anti-mouse secondary antibody, and visualised using enhanced chemiluminescence detection. The expression level of GAPDH was used as the loading control for the total protein. The antibodies used in this study included anti-phospho-

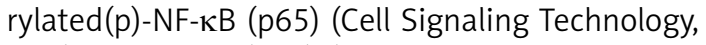
USA), anti-NF-кB(p65) (Cell Signaling Technology,
USA), anti-CD9 (Abcam, UK), anti-CD63 (Abcam, UK), and anti-GAPDH (Proteintech, USA). Antigen-antibody complexes were detected using secondary antibodies conjugated to HRP (Invitrogen, USA) and visualised through enhanced chemiluminescence (Biological Industries, IL). Each western blot was independently repeated at least three times, and representative results are shown.

\section{Reverse transcription PCR (RT-PCR) and quantitative real-time PCR analysis}

To analyse the RNA expression levels of the macrophages, qRT-PCR was performed. For RT-PCR, total RNA was extracted using Trizol and was spectrophotometrically quantified, and equal amounts $(1 \mu \mathrm{g})$ were transcribed into cDNA according to the instructions. mRNA levels were quantified by qRT$P C R$ using the Syber green master mix (Vazyme, JS, CHN). The data analyses were performed using the $2^{-\Delta \Delta C t}$ method, whereby relative gene expression levels were normalised to GAPDH and mRNA abundance was quantified using the threshold cycle method. CD163, CD206, IL-10, IL-6, IL-12, and CXCL10 mRNA expression in macrophages were assessed. The primer sequences for the molecules used were listed as follows. Sense and antisense primers for CD163 (ATCAACCCTGCATCTTTAGACA; CCTGTTGTCACATGTGATCCAG), CD206 (GACGTGGCTGTGGATAAATAAC; CAGAAGACGCATGTAAAGCTAC), IL-10 (GTTGTTAAAGGAGTCCTTGCTG; TTCACAGGGAAGAAATCGATGA), IL-6 (CACTGGTCTTTTGGAGTTTGAG; GGACTTTTFTACTCATCTGCAC), IL-12 (TAAGATGCGAGGCCAAGAATTA; TACTCATACTCCTTGTTGTTCC), CXCL10 (CTCTCTCTAGAACTGTACGCTG; AATCAGACATCTCTTCTCACCC), as well as GAPDH (CAGGAGGCATTGCTGATGAT; GAAGGCTGGGGCTCATTT), were used for qRT-PCR analysis. Experiments were completed in biological triplicate, and results are expressed in terms of fold change using GAPDH as the housekeeping gene.

\section{Statistical analysis}

All data are presented as mean \pm SD of results obtained from at least three independent experiments. Graph generation and statistical analyses were performed using GraphPad Prism software (GraphPad Software, Inc., La Jolla, CA, USA). Statistical significance was assessed using unpaired Student's t-tests, with $p<0.05$ considered statistically significant. ${ }^{*} p<0.05,{ }^{* *} p<0.01$, and ${ }^{* * *} p<0.001$ are indicated in the figure legends.

\section{Results}

\section{Morphology and characterisation of lymphoma exosomes}

OCI-LY1, OCI-LY3, and SU-DHL8 cells were cultured in exosome-free cell media, and the super- 
natants were collected. Exosomes isolated and purified from the supernatants were visualised using TEM, which presented cup-shaped membrane vesicles. There was no significant difference in the morphology of the exosomes isolated from the three cell types (Figure $1 \mathrm{~A}$ ). Marker proteins of OCI-LY1 and OCI-LY3-derived exosomes were detected, including the expression of CD9 and CD63. Western blot results showed that marker proteins of exosomes from the two cell lines could be detected, and extracted exosomes were further identified at the molecular level (Figure 1 B). The results showed that exosomes from different DLBCL subtype cells were successfully isolated.

\section{Exosomes from lymphoma cells regulate macrophage polarisation}

The polarisation of macrophages is easily influenced by the TME; we hypothesise that exosomes derived from lymphoma cell lines could acclimate THP-1-derived macrophages to an immunosuppressive phenotype, contributing to tumour cell progression. Many previous studies have shown that IL-10 is a key factor in the polarisation of M2 macrophages [18-23]. Under indirect co-culture conditions, we evaluated the mRNA expression of M2-related polarisation markers CD163, CD206, and IL-10 in macrophages of different stages. Then we co-cultured different DLBCL cells subtypes OCI$\mathrm{LY} 1$ and $\mathrm{OCl}-\mathrm{LY} 3$ with THP-1-derived $\mathrm{MO}$ or M2 macrophages, and the macrophages of each phenotype were divided into an experimental group and a control group - the experimental group were treated with different DLBCL exosomes for
48 h. After co-culture, the mRNA expression of CD163, CD206, and IL-10 in macrophages of different phenotypes was detected via quantitative real-time PCR. We found that the expression levels of CD163, CD206, and IL-10 were significantly upregulated in $\mathrm{MO}$ macrophages treated with lymphoma exosomes (Figures $2 \mathrm{~A}, \mathrm{~B}$ ), while the expression levels of CD163, CD206, and IL-10 were not upregulated in M2 macrophages treated with lymphoma exosomes (Figures 2 C, D).

\section{Uptake of DiO-labelled DLBCL-derived exosomes}

To investigate the uptake of DLBCL-derived exosomes by macrophages, we evaluated whether DLBCL-exosomes are efficiently internalised into macrophages by using laser scanning confocal microscopy. Both DiO-labelled OCI-LY3- and OCI-LY1-derived exosomes were incorporated into macrophages of different stages (Figures $3 \mathrm{~A}, \mathrm{~B}$ ). These data suggest that exosomes derived from DLBCL cells can be internalised into THP-1-derived macrophages.

\section{The effect of exosome-treated macrophages on apoptosis of DLBCL cells}

To identify the impact of macrophages treated with exosomes on DLBCL cell apoptosis, early and late apoptosis was assessed by Annexin V-FITC/ $\mathrm{PI}$ double staining. We found that in the indirect co-culture of $\mathrm{MO}$ macrophages and different DLBCL subtype cells, in the group of macrophages treated with different DLBCL exosomes, the drug-induced apoptosis of corresponding DLBCL
A

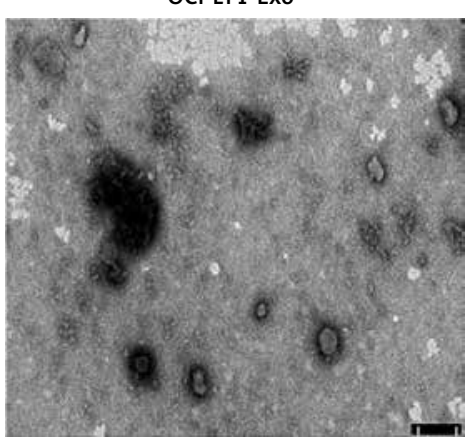

OCI-LY3-Exo

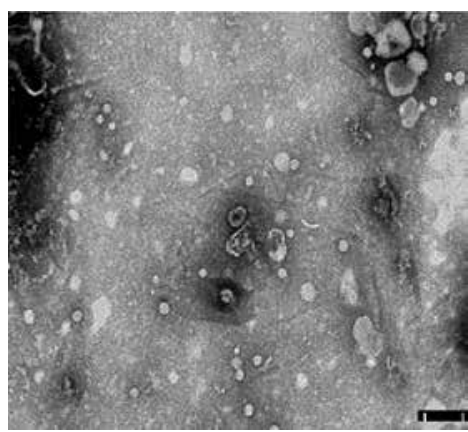

SU-DHL8-Exo

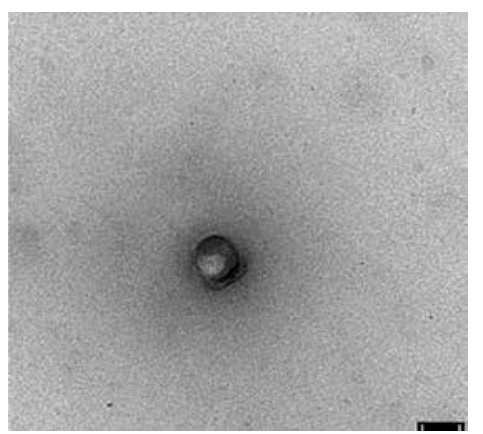

B
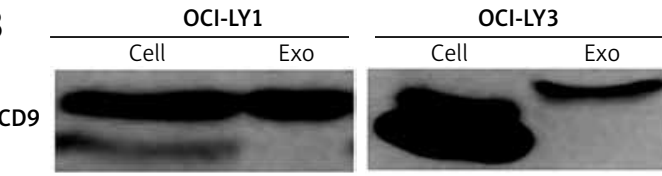

CD63
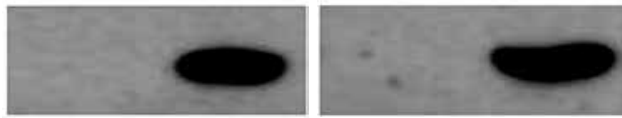

Figure 1. Morphology and characterization of lymphoma exosomes. A - Transmission electron microscopy of isolated exosomes indicates a cupshaped structure. The size bar represents $50 \mathrm{~nm}$. The diameter ranged from 30-150 nm. B - Expression of exosome and cell marker proteins CD9 and CD63 in OCI-LY1 and OCI-LY3 cell lysate (cell) and exosomes (Exo) 

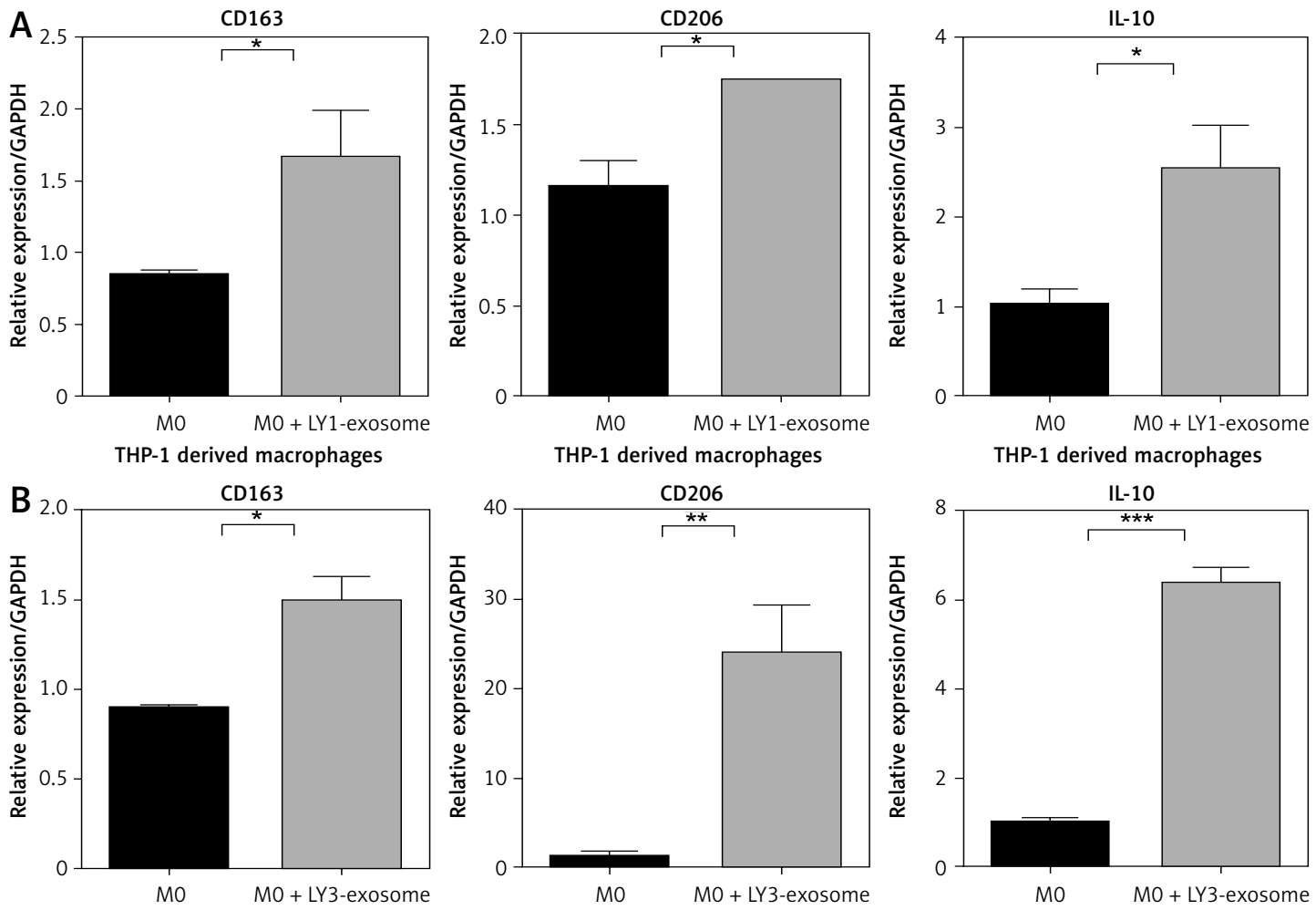

THP-1 derived macrophages

THP-1 derived macrophages

THP-1 derived macrophages
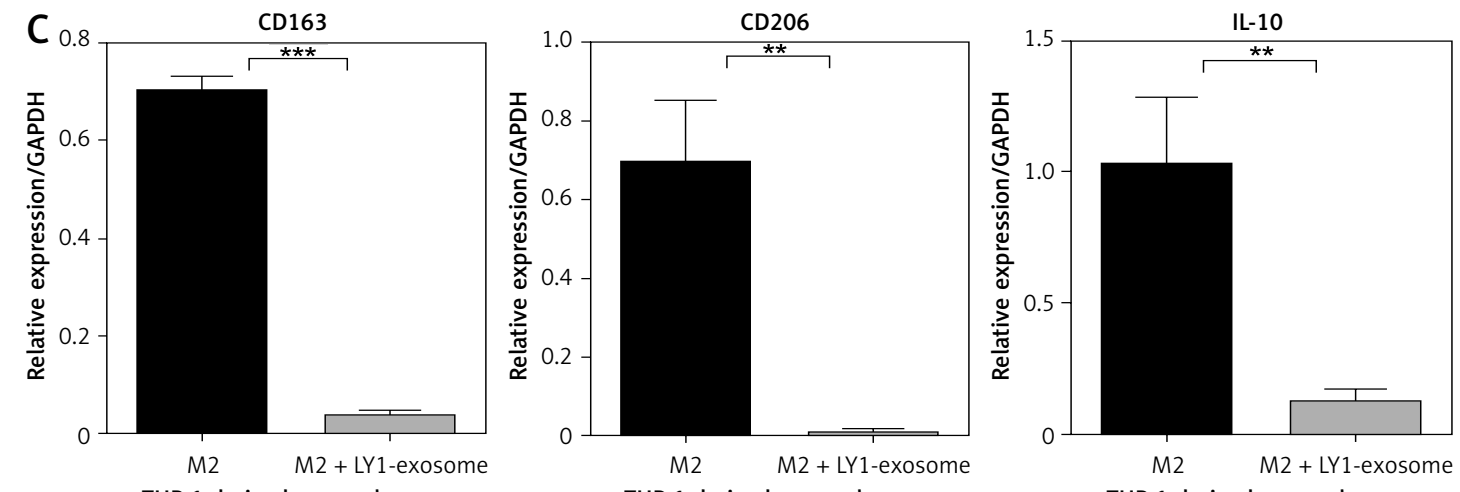

THP-1 derived macrophages

THP-1 derived macrophages

M2 M2 + LY1-exosome
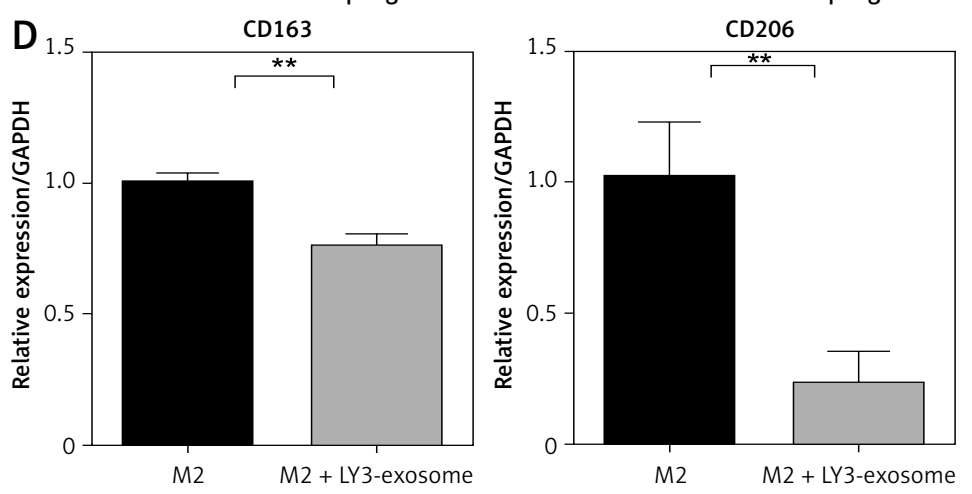

THP-1 derived macrophages

$\mathrm{M} 2$

$\mathrm{M} 2+$ LY3-exosome THP-1 derived macrophages
THP-1 derived macrophages

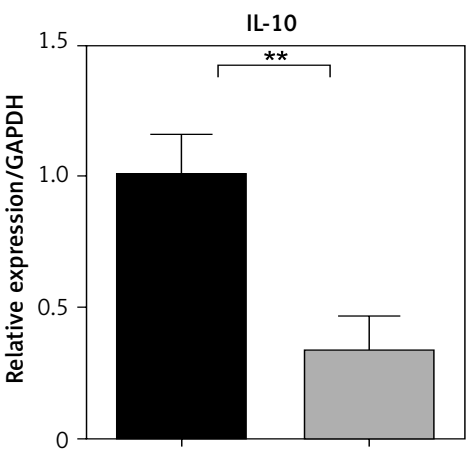

M2 THP-1 derived macrophages

Figure 2. Exosomes from lymphoma cells OCI-LY1 and OCI-LY3 regulate macrophage polarization in co-culture. A - The expression of CD163, CD206, and IL-10 in THP-1-derived MO macrophages treated or untreated with OCI-LY1-secreted exosomes. B - The expression of CD163, CD206, and IL-10 in THP-1-derived MO macrophages treated or untreated with OCI-LY3-secreted exosomes. C - The expression of CD163, CD206, and IL-10 in THP-1derived M2 macrophages treated or untreated with OCI-LY1-secreted exosomes. D - The expression of CD163, CD206, and IL-10 in THP-1-derived M2 macrophages treated or untreated with OCI-LY3-secreted exosomes. Results are shown as relative to PBS-treated control $(n=3)$

${ }^{\star} P<0.05 ;{ }^{* *} p<0.01 ;{ }^{* \star *} p<0.001$. 

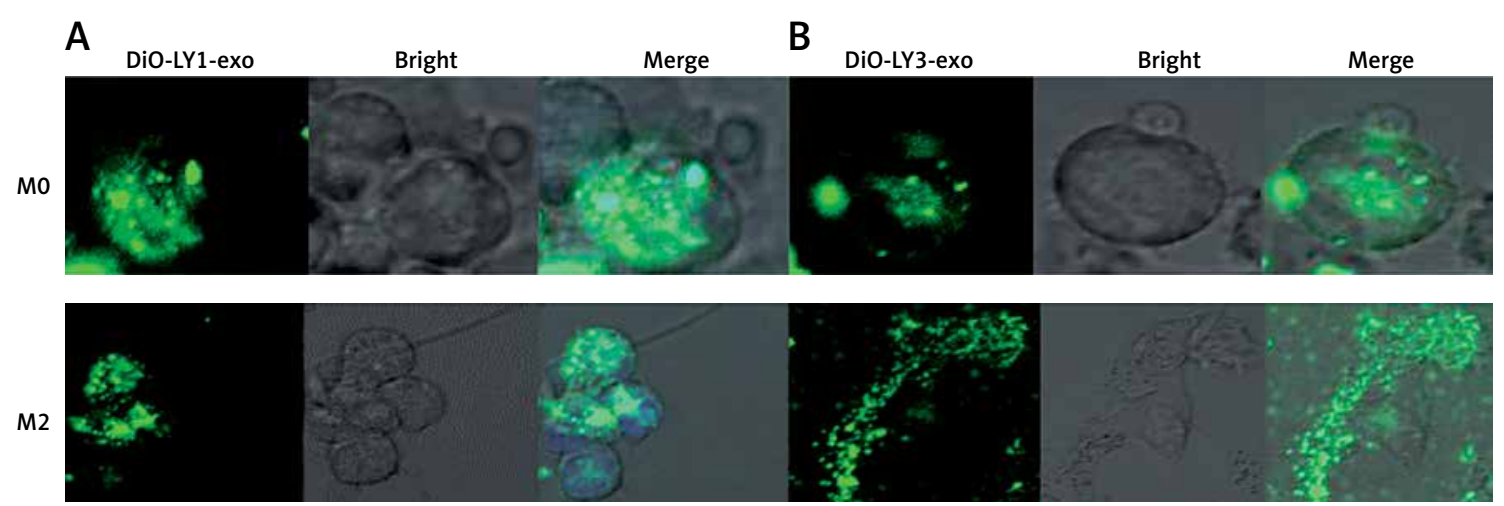

Figure 3. Exosomes derived from OCI-LY1 and OCI-LY3 cells are taken up by THP-1 derived macrophages (MO and M2). A - Representative confocal microscopy images at 40× magnification, showing the uptake of DiO-labeled OClLY1-derived exosomes by macrophages (MO and M2) after $4 \mathrm{~h}$ incubation. B - Representative confocal microscopy images at 40x magnification, showing the uptake of DiO-labeled OCl-LY3-derived exosomes by macrophages ( $\mathrm{MO}$ and $\mathrm{M} 2$ ) after $4 \mathrm{~h}$ incubation

cells decreased (Figures 4 A, B). However, in the indirect co-culture of $M 2$ macrophages and different DLBCL subtype cells, in the group of macrophages treated with the combination of lymphoma exosomes and IL-4/IL-13, the drug-induced apoptosis of lymphoma cells increased (Figures 4 C, D). We found that macrophages of different stages treated with lymphoma exosomes had different effects on the drug-induced apoptosis of lymphoma cells under the indirect co-culture conditions.

\section{The effect of different DLBCL-derived} exosomes on p65(NF- $\mathrm{kB}$ ) phosphorylation in macrophages

To investigate the effect of lymphoma exosomes on the NF- $\mathrm{KB}$ signalling pathway in macrophages, we cultured THP-1-derived MO macrophages with $150 \mu \mathrm{g} / \mathrm{ml}$ lymphoma exosomes, and we detected the protein expression of phosphorylation p65 and total NF- $\kappa B(p 65)$ in these macrophages treated with OCI-LY1 exosomes. We found that the expression of p-p65 in M0 macrophages treated with $\mathrm{OCl}-\mathrm{LY} 1$ exosomes was significantly upregulated (Figure $5 \mathrm{~A}$ ), while the protein expression of total NF- $\mathrm{KB}(\mathrm{p} 65)$ was not significantly altered. At the same time, we also detected the $\mathrm{NF}-\kappa \mathrm{B}$ signalling pathway activated in $\mathrm{M} 2$ macrophages treated with OCl-LY1 exosomes, and found that the protein expression of p-p65 was also significantly upregulated, while total NF- $\mathrm{KB}(\mathrm{p} 65)$ did not change (Figure $5 \mathrm{~B}$ ). In addition, the mRNA expression of related inflammatory factors downstream of NF- $\mathrm{KB}$ signalling was screened, and the expression of IL-12, IL-6, and CXCL10 was significantly upregulated (Figures $5 \mathrm{E}, \mathrm{F}$ ).

To further confirm the effect of DLBCL exosomes on the NF- $\mathrm{KB}$ signalling pathway in macrophages, we used OCl-LY3-derived exosomes on THP-1 derived macrophages of different stages. $\mathrm{OCl}-\mathrm{LY} 3$-derived exosomes were added to THP-1- derived MO macrophages, and western blot analysis evaluated the protein expression of p-p65 and total NF- $\mathrm{KB}(\mathrm{p} 65)$ in these cells. We found that the protein expression of p-p65 in MO macrophages treated with $\mathrm{OCl}-\mathrm{LY} 3$ exosomes was not upregulated, and the protein expression of total NF- $\kappa B(p 65)$ did not change significantly (Figure $6 \mathrm{~A})$. However, in $\mathrm{M} 2$ macrophages treated with $\mathrm{OCl}-\mathrm{LY} 3$ exosomes, we found that the protein expression of p-p65 was significantly upregulated, and total NF- $\mathrm{B}(\mathrm{p} 65)$ protein expression did not change (Figure $6 \mathrm{~B}$ ). The mRNA expression of inflammatory cytokines downstream of NF- $\kappa B$ signalling were also examined, and it was found that the mRNA expression of IL-12, IL-6, and CXCL10 was significantly upregulated (Figures 6 E, F) in macrophages of different stages treated with DL$\mathrm{BCL}$ exosomes. This indicated that different $\mathrm{DLBCL}$ exosomes have different effects on activation of p-p65 in macrophages of different stages in the TME.

\section{Discussion}

Exosomes are bioactive vesicles that contain abundant bioactive substance and play a key role in the regulation of intercellular signal transduction [24, 25]. Tumour-derived exosomes are an efficient platform for the transfer and exchange of cellular contents between tumours and surrounding cells in the TME, and they play a key role in regulating the TME function [26]. In this study, differential centrifugation, together with a commercial kit, was used to isolate exosomes. A high concentration of isolated and purified exosomes was used for biological function assays. Currently, CD9, CD63, CD81, and Hsp70 are commonly used to identify exosomes. Here, CD9 and CD63 were detected on the surface of isolated and purified DLBCL-derived exosomes, confirming the successful extraction of tumour exosomes. 
A

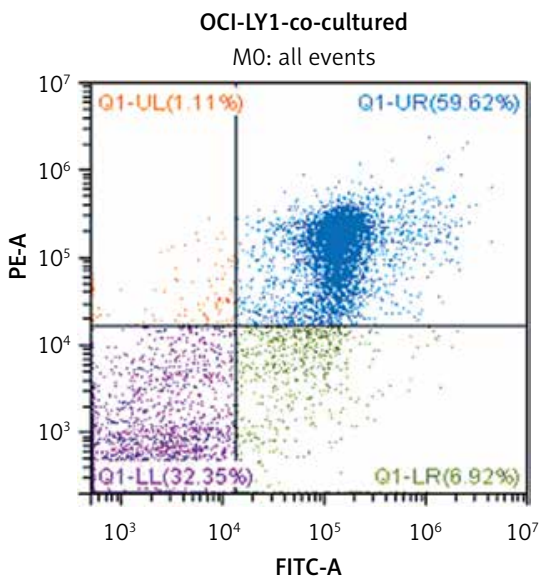

OCI-LY1-co-cultured

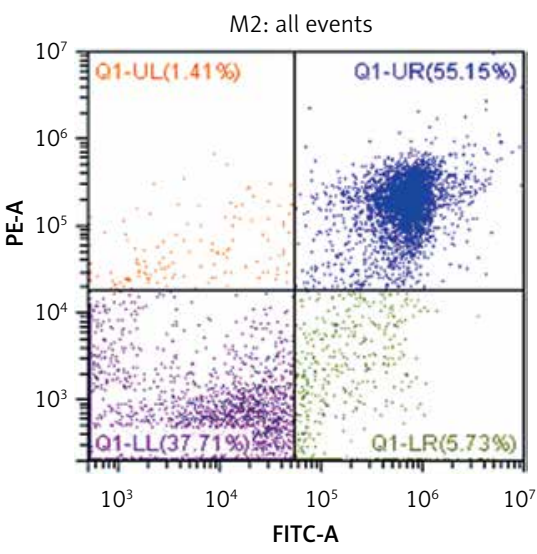

OCI-LY3-co-cultured

C

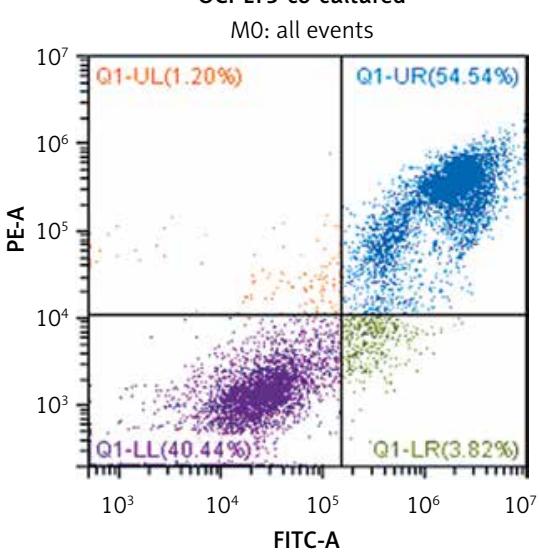

OCI-LY1-co-cultured

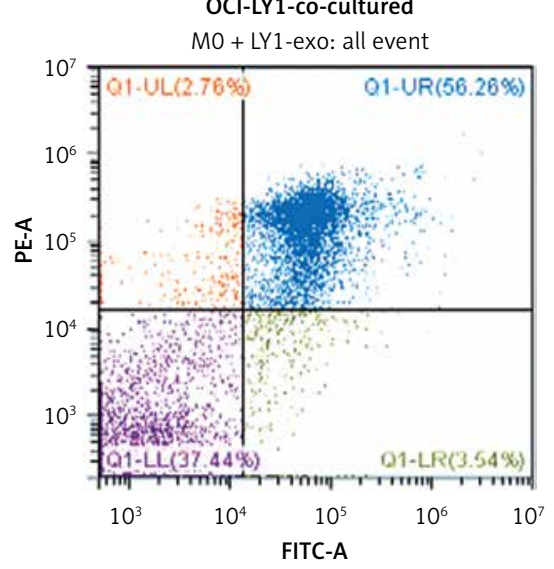

OCI-LY1-co-cultured

M2 + LY1-exo: all events

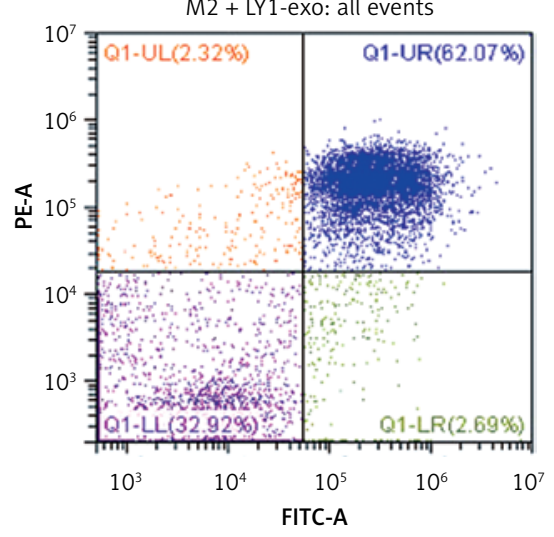

OCI-LY3-co-cultured

MO + LY3-exo: all event

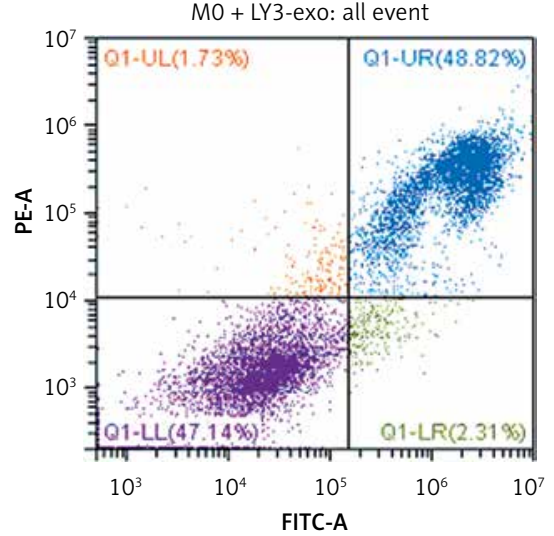

Figure 4. Effect of macrophages treated with exosomes on apoptosis of DLBCL cells. A-OCI-LY1 cells were incubated in the upper transwell chamber and indirectly co-cultured with $\mathrm{MO}$ macrophages treated with OCl-LY1-derived exosomes under the chamber for $24 \mathrm{~h}$, before epirubicin was added for $24 \mathrm{~h}$. The cells were analyzed by FCM. B - OCl-LY1 cells were incubated in the upper transwell chamber and indirectly co-cultured with M2 macrophages treated with OCI-LY1-derived exosomes under the chamber for $24 \mathrm{~h}$, before epirubicin was added for $24 \mathrm{~h}$. The cells were analyzed by FCM. C - OCl-LY3 cells were incubated in the upper transwell chamber and indirectly co-cultured with $\mathrm{MO}$ macrophages treated with $\mathrm{OCl}-\mathrm{LY} 3$-derived exosomes under the chamber for $24 \mathrm{~h}$, before epirubicin was added for $24 \mathrm{~h}$ 
D

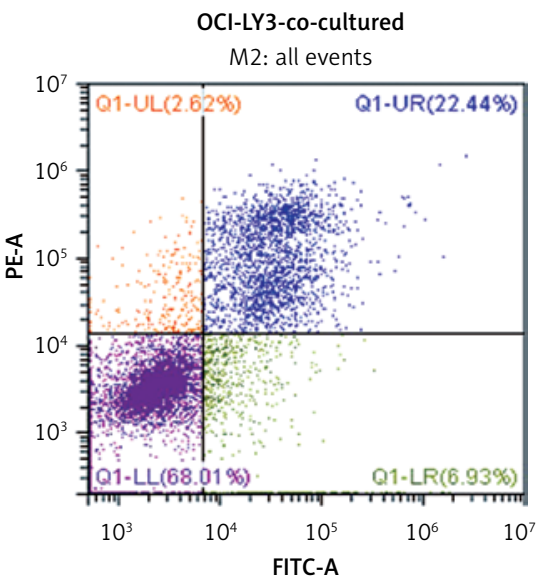

E

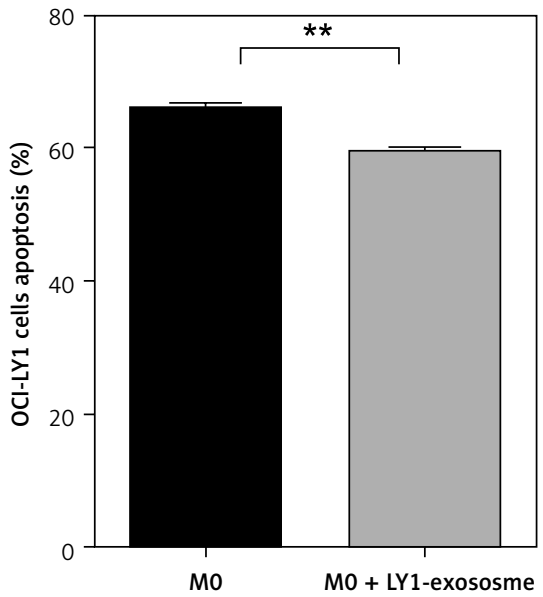

G

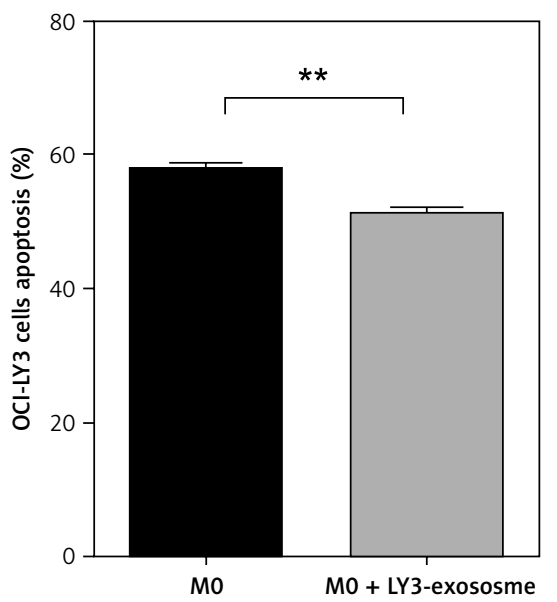

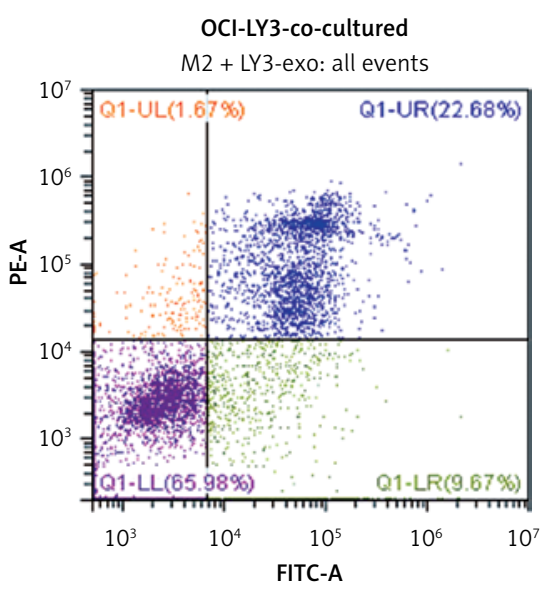

$\mathrm{F}$

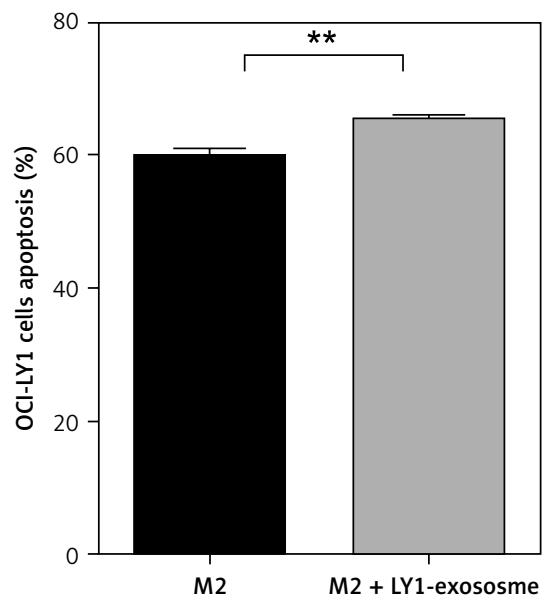

$\mathrm{H}$

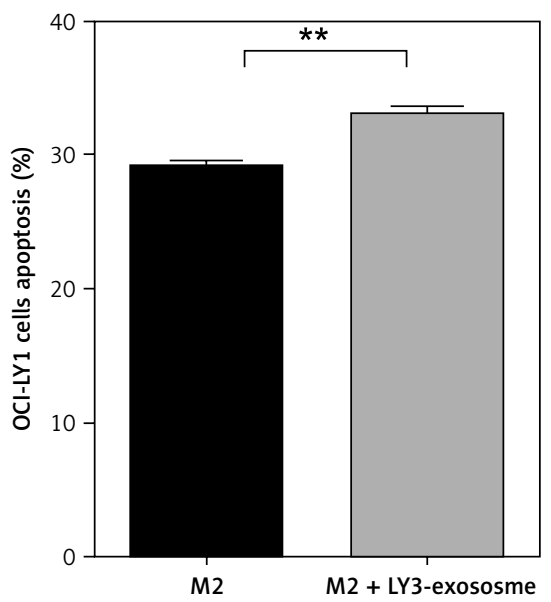

Figure 4. Cont. D- OCI-LY3 cells were incubated in the upper transwell chamber and indirectly co-cultured with $\mathrm{M} 2$ macrophages treated with OCl-LY3-derived exosomes under the chamber for $24 \mathrm{~h}$, before epirubicin was added for $24 \mathrm{~h}$. The cells were analyzed by FCM. The histograms of $(\mathbf{E}),(\mathbf{F}),(\mathbf{G})$, and $(\mathrm{H})$ represents the data of $(\mathbf{A}),(\mathrm{B}),(\mathbf{C})$, and (D), respectively. Results are presented relative to control $(n=3)$

${ }^{*} p<0.05 ;{ }^{* *} p<0.01 ;{ }^{* * *} p<0.001$.

Within the cancer microenvironment, immune cells often display altered phenotypes that contribute to tumour progression through promotion of tumour growth, migration, pre-metastatic niche formation, and metastasis [27, 28]. Macrophages are important immune cells in the TME and play an important role in the formation of the inflam- matory microenvironment and the development of tumours. The pro-tumour response is induced in immune cells by following direct or indirect stimulation of cancer cells in the TME, and one potential mechanism could be through the action of tumour-derived exosomes [26]. Exosomes carry a variety of genetic material, proteins, and lip- 

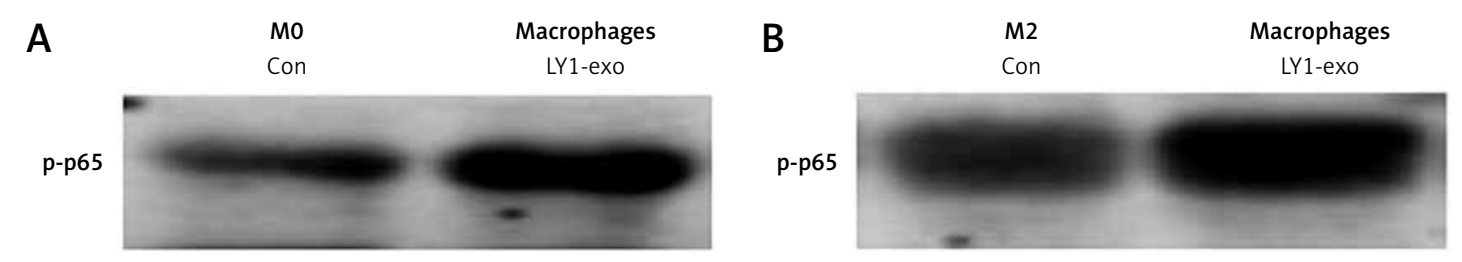

p65

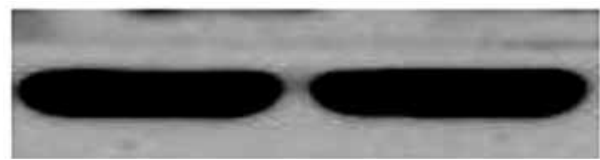

p65

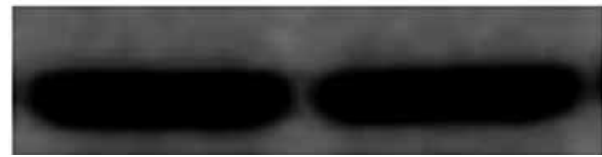

GAPDH

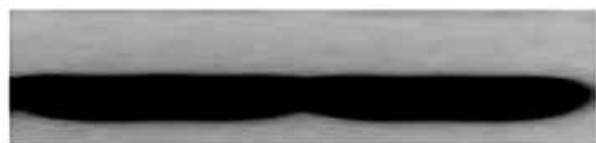

GAPDH

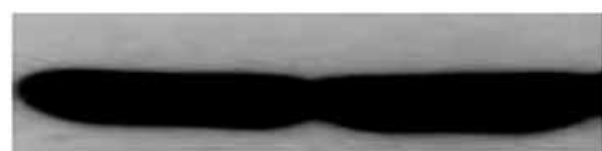

C

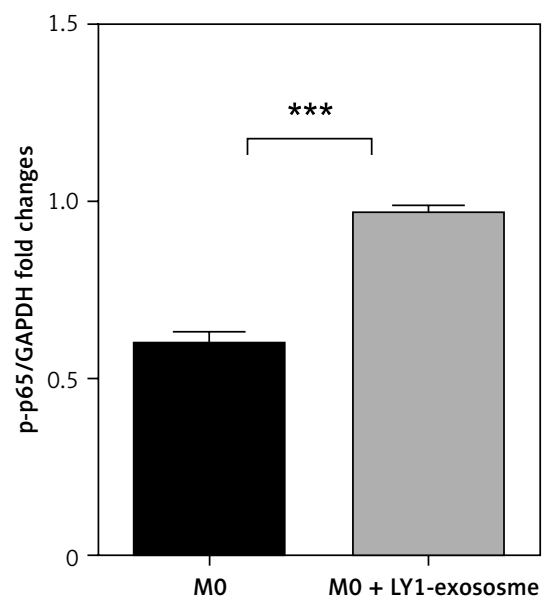

D

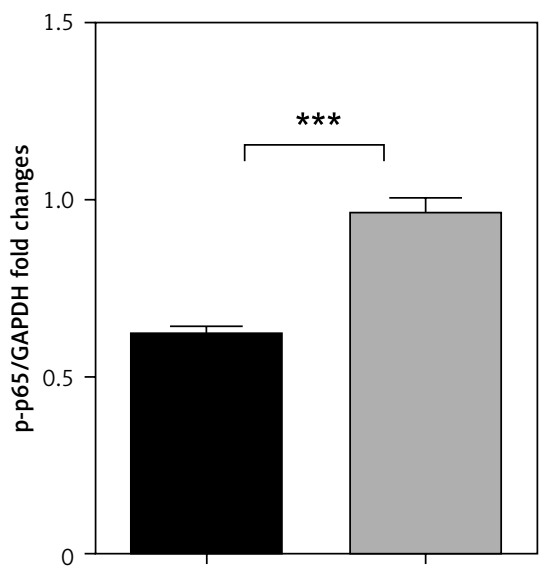

M2

M2 + LY1-exososme
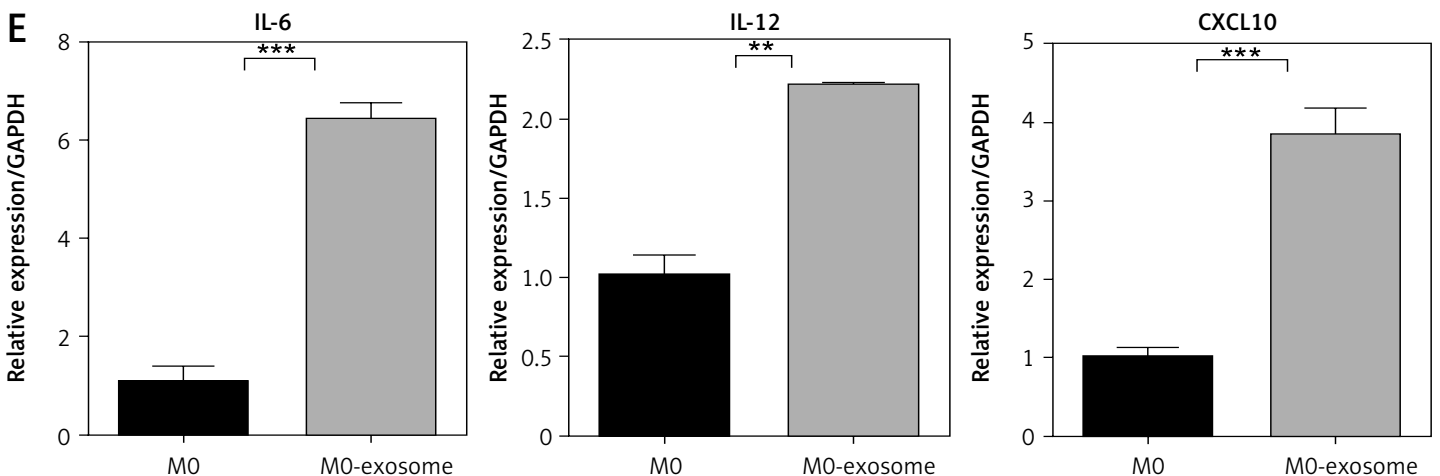

THP-1-derived macrophages

THP-1-derived macrophages

THP-1-derived macrophages

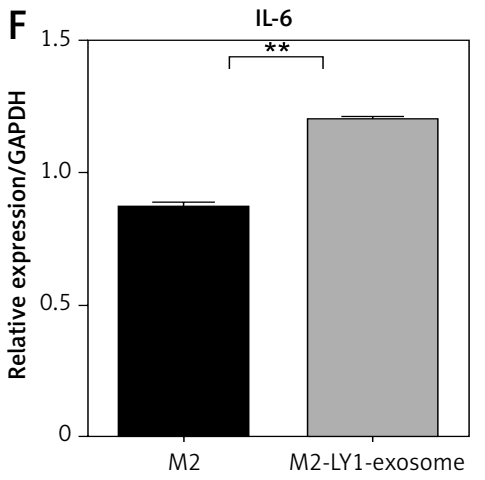

THP-1-derived macrophages

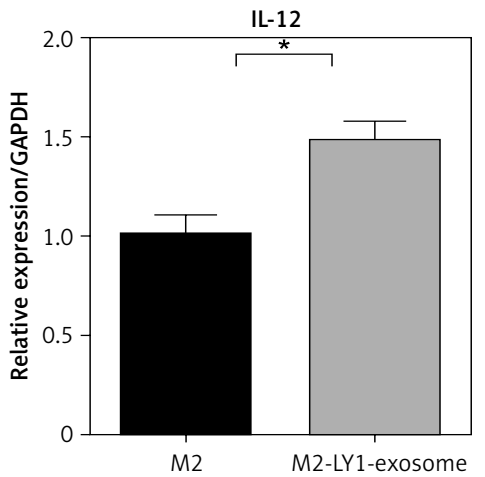

THP-1-derived macrophages

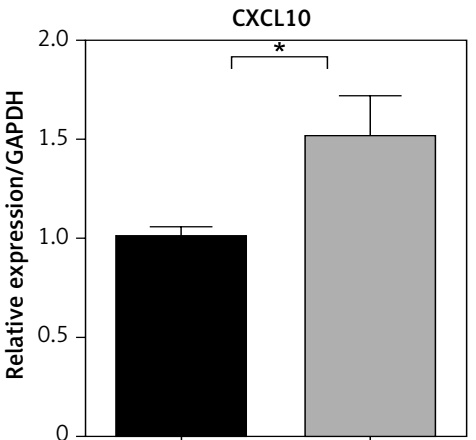

$\mathrm{M} 2$

M2-LY1-exosome

THP-1-derived macrophages

Figure 5. Effect of the subtype of DLBCL OCI-LY1-derived exosomes on phosphorylation of p65 and mRNA expression of IL-12, IL-6, and CXCL10 in macrophages. A - Expression of p-p65 in M0 macrophages treated with OCI-LY1 exosomes. B - Expression of p-p65 in M2 macrophages treated with OCI-LY1 exosomes combined with IL-4/IL-13. C - Densitometric analysis of p-p65 levels in MO macrophages treated with OCI-LY1 exosomes, normalized to GAPDH. D - Densitometric analysis of p-p65 levels in M2 macrophages treated with OCl-LY1 exosomes combined with IL-4/IL-13, normalized to GAPDH. E - mRNA expression of IL-12, IL-6, and CXCL10 in M0 macrophages treated with OCI-LY1 exosomes. F - mRNA expression of IL-12, IL-6, and CXCL10 in M2 macrophages treated with OCI-LY1 exosomes combined with IL-4/IL-13. Results are relative to the control $(n=3)$

${ }^{*} p<0.05 ;{ }^{* *} p<0.01 ;{ }^{* * *} p<0.001$. 
A

p-p65

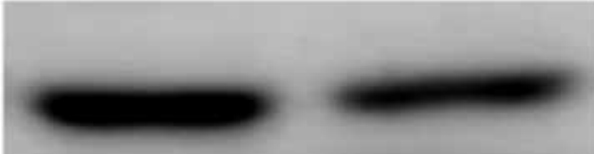

p65

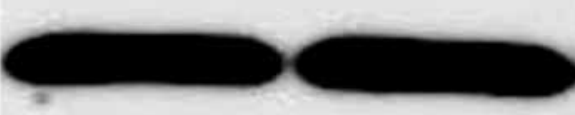

GAPDH

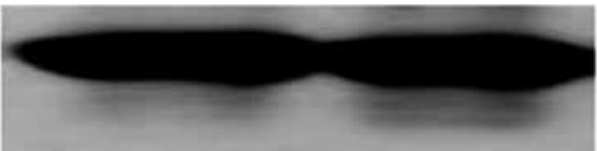

C

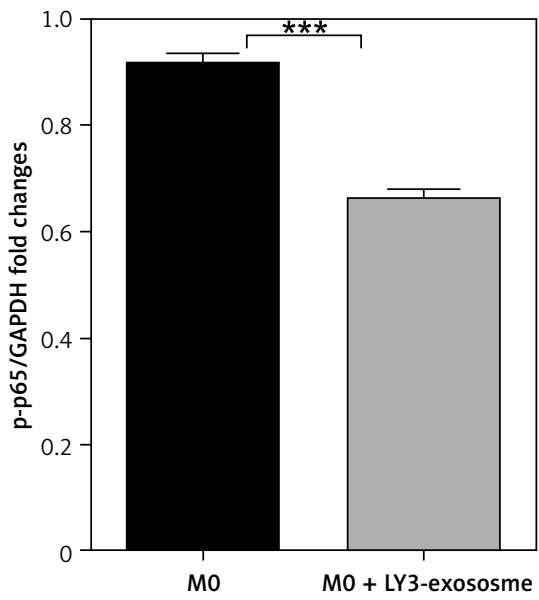

B

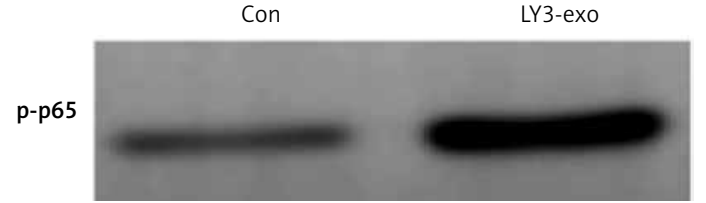

p6

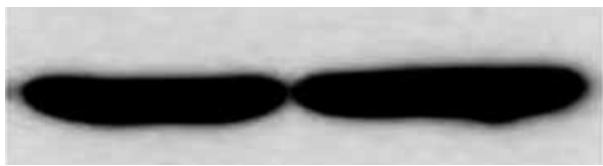

GAPDH

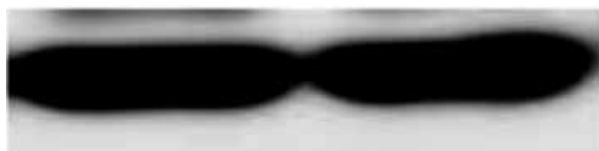

D

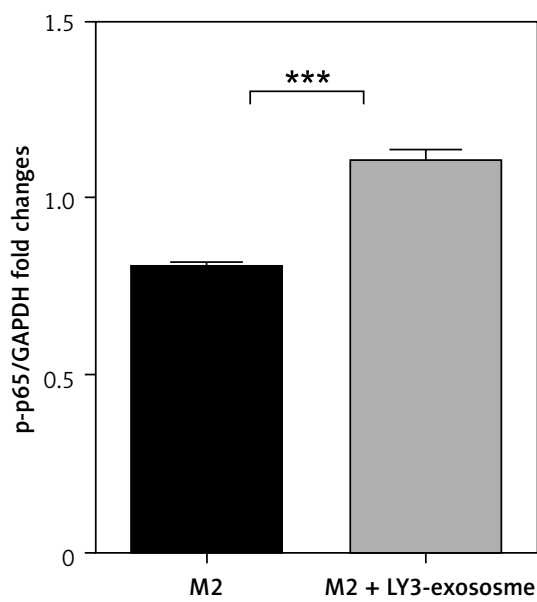

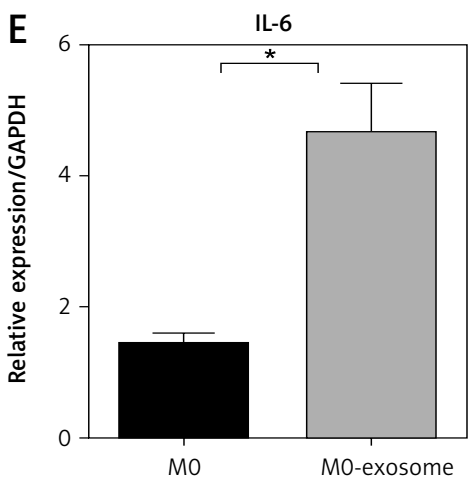

THP-1-derived macrophages

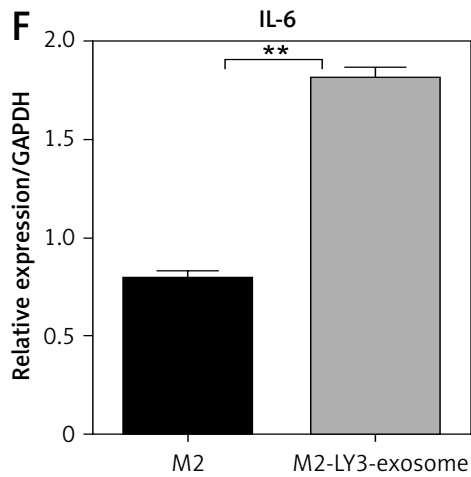

THP-1-derived macrophages

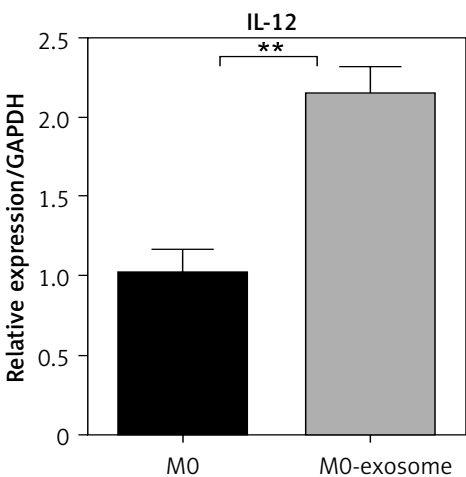

THP-1-derived macrophages

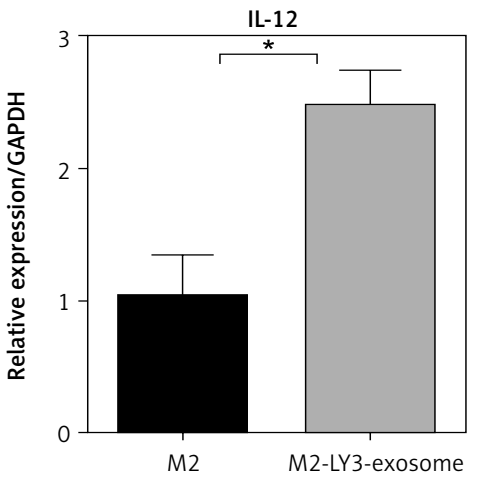

THP-1-derived macrophages

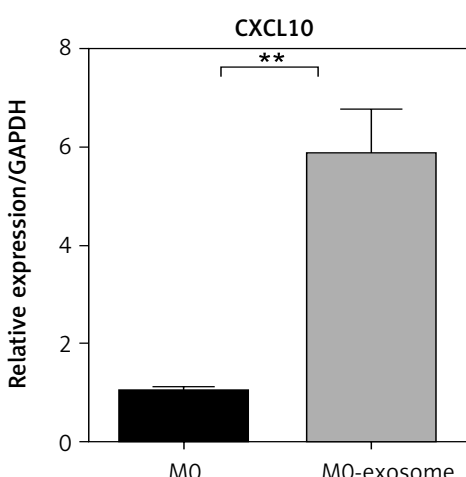

THP-1-derived macrophages

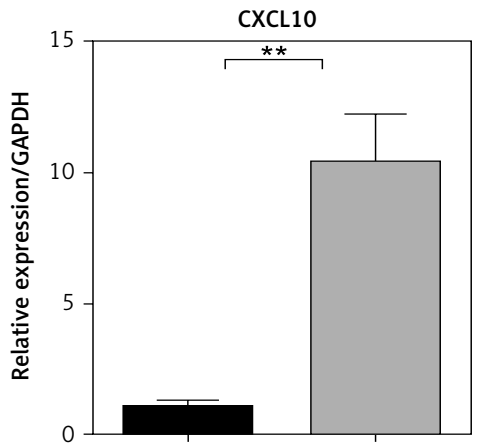

$\mathrm{M} 2$

M2-LY3-exosome

THP-1-derived macrophages

Figure 6. Effect of the subtype of DLBCL OCI-LY3-derived exosomes on phosphorylation of p65 and mRNA expression of IL-12, IL-6, and CXCL10 in macrophages. A - Expression of p-p65 in M0 macrophages treated with OCI-LY3 exosomes. B - Expression of p-p65 in M2 macrophages treated with OCI-LY3 exosomes combined with IL-4/IL-13. C - Densitometric analysis of p-p65 levels in MO macrophages treated with OCI-LY3 exosomes, normalized to GAPDH. D - Densitometric analysis of p-p65 levels in M2 macrophages treated with OCI-LY3 exosomes combined with IL-4/IL-13, normalized to GAPDH. E - mRNA expression of IL-12, IL-6, and CXCL10 in M0 macrophages treated with OCI-LY3 exosomes. F - mRNA expression of IL-12, IL-6, and CXCL10 in M2 macrophages treated with OCI-LY3 exosomes combined with IL-4/IL-13. Results are relative to the control $(n=3)$

${ }^{*} p<0.05 ;{ }^{* *} p<0.01$; ${ }^{* * *} p<0.001$. 
ids, all of which may functionally alter recipient cell phenotypes [29]. Aung et al. indicated that B-cell lymphoma cell-derived exosomes could transfer CD20, which combined with therapeutic anti-CD20 antibodies protecting target cells from antibody attack [30]. The dynamic interaction between tumours and TAMs coordinates key events leading to tumourigenesis and drug-resistance [31-33]. However, in the research field of DLBCL, the underlying mechanisms of macrophages promoting the progression of lymphoma in the TME are unclear.

Herein, we have confirmed that exosomes derived from DLBCL cells can be internalised by macrophages in the TME with high efficiency. Next, we investigated the role exosomes play in macrophages after they are internalised.

DLBCL-derived exosomes were collected to act on macrophages, which were indirectly co-cultured with corresponding lymphoma cells for $48 \mathrm{~h}$, and then the chemotherapy drug epirubicin was added to these lymphoma cells and incubated for $24 \mathrm{~h}$. Then, we assessed the growth and anti-apoptotic ability of DLBCL cells through FCM. We found that inactive $M 0$ macrophages treated with $D L B C L$ exosomes can enhance the resistance of lymphoma cells to drug-induced apoptosis and promote the anti-apoptotic ability of lymphoma cells. However, for the M2 macrophages in the polarisation stage, treatment with DLBCL exosomes enhanced the drug-induced apoptosis of lymphoma cells and did not promote the anti-apoptotic ability of lymphoma cells. Therefore, we believe that in the TME, DLBCL-derived exosomes have different effects on macrophages of different stages, thus having different effects on the drug-induced apoptosis of lymphoma. Related assays were conducted to assess the role of macrophages treated with exosomes on the drug-resistance of lymphoma; these showed that inactive MO macrophages that internalise exosomes, whether from GCB subtype OCI-LY1 or non-GCB subtype OCI-LY3 cells, can promote drug-resistance in lymphoma. This study provides important experimental support demonstrating that inactive MO macrophages treated with exosomes increases the drug-resistance of lymphoma cells, which is vital for future research into the potential underlying mechanisms of macrophage drug-resistance in lymphoma.

The development of lymphoma is a very complicated process. Many studies suggest that the TME plays a key role in the formation of heterogeneity of lymphoma cells, and an immunosuppressed microenvironment can promote the growth, metastasis, and invasive ability of tumour cells [2, 3]. The composition of immune cells is complicated, and macrophages are the main component [34]. We focused on the fundamental NF-кB pathway, which is critical in the development of cancer, and the expression of $\mathrm{p}-\mathrm{p} 65$ and $\mathrm{NF}-\mathrm{\kappa B}(\mathrm{p} 65)$ protein in different stages of macrophages treated with DLBCL-derived exosomes. We found that in GCB subtype OCI-LY1-derived exosomes applied to MO macrophages, the expression of intracellular p-p65 protein clearly increased, while the total expression of NF- $\mathrm{KB}(\mathrm{p} 65)$ protein showed no obvious change. When OCl-LY1-derived exosomes were introduced to polarising $\mathrm{M} 2$ macrophages, the expression of intracellular p-p65 protein was similarly upregulated while the total expression of NF- $\kappa \mathrm{B}(\mathrm{p} 65)$ protein showed no obvious change. The trend of $p$-p65 protein expression in macrophages at different stages treated with OCI-LY1derived exosomes is consistent. Together, the activation of NF- $\kappa B$ signalling in macrophages of different stages treated with $\mathrm{OCl}-\mathrm{LY} 1$-derived exosomes is at least partially induced by tumour exosomes.

Additionally, we verified the effect of another non-GCB subtype of DLBCL cells lines; OCI-LY3-derived exosomes on macrophages in the TME were investigated. We found that when $\mathrm{OCl}-\mathrm{LY} 3$-derived exosomes were applied to MO macrophages, the expression of intracellular $\mathrm{p}$-p65 protein did not increase, while the total expression of NF- $\kappa \mathrm{B}(\mathrm{p} 65)$ protein showed no obvious change. When $\mathrm{OCl}$ LY3-derived exosomes were applied to polarising M2 macrophages, the expression of intracellular p-p65 protein was upregulated, while the total expression of NF- $\mathrm{BB}(\mathrm{p} 65)$ showed no change. The trend of $\mathrm{p}-\mathrm{p} 65$ protein expression in macrophages at different stages treated with $\mathrm{OCl}-\mathrm{LY} 3$-derived exosomes is inconsistent. Taken together, the activation of NF- $\mathrm{KB}$ in macrophages of different stages treated with OCl-LY3-derived exosomes was different. In this study, inflammatory factors in downstream NF- $\kappa$ B signalling pathway were also assessed. The mRNA expression of IL-6, IL-12, and CXCL10 was upregulated in macrophages treated with different subtypes of DLBCL-derived exosomes. These data suggest that the activation of NF- $\mathrm{KB}$ in inactivated $\mathrm{MO}$ macrophages treated with exosomes from different subtypes of DLB$\mathrm{CL}$ cells is different. Therefore, NF-KB signalling is not the only signalling pathway for lymphoma exosomes activating MO macrophages to release more inflammatory factors. However, for the polarising M2 macrophages, lymphoma exosomes from different subtypes of DLBCL cells can induce the activation of NF- $\mathrm{KB}$ signalling in macrophages to release more inflammatory cytokines.

In conclusion, this study shows that macrophages treated with DLBCL exosomes can produce more proinflammatory cytokines (IL-6, IL-12, and CXCL10), but exosomes from different subtypes of DLBCL cells have different effects on the acti- 
vation of NF- $\kappa B$ signalling in macrophages at different stages. Exosomes from the GCB subtype of DLBCL cells can activate NF- $\kappa B$ in macrophages at different stages, while exosomes from non-GCB subtype DLBCL cells only activate NF- $\kappa B$ signalling in $M 2$ macrophages that are in a phase of polarisation. Our study was limited to the whole exosome. The next step is to study how specific contents of exosomes affect macrophage polarisation. Altogether, exosomes from different subtypes of DLBCL cells can activate macrophages in the microenvironment to produce more inflammatory cytokines, but the mechanisms regarding the activation of NF- $\mathrm{KB}$ signalling in different macrophage stages is different. This provides new evidence in the study of exosome-mediated activation of macrophages in the TME and presents new ideas for clinical intervention and treatment of different subtypes of DLBCL.

\section{Acknowledgments}

Huayu Ling, Zhong Yang - equal contribution.

\section{Conflict of interest}

The authors declare no conflict of interest.

\section{References}

1. Friedberg JW. New strategies in diffuse large B-cell lymphoma: translating findings from gene expression analyses into clinical practice. Clin Cancer Res 2012; 17: 6112-7.

2. Coussens LM, Werb Z. Inflammation and cancer. Nature 2002; 420: 860-7.

3. Scott DW, Gascoyne RD. The tumour microenvironment in B cell lymphomas. Nat Rev Cancer 2014; 14: 517-34.

4. Murray PJ, Wynn TA. Protective and pathogenic functions of macrophage subsets. Nat Rev Immunol 2011; 11: 723-37.

5. Shi C, Pamer EG. Monocyte recruitment during infection and inflammation. Nat Rev Immunol 2011; 11: 762-74.

6. Qian BZ, Pollard JW. Macrophage diversity enhances tumor progression and metastasis. Cell 2010; 141: 39-51.

7. Biswas SK, Sica A, Lewis CE. Plasticity of macrophage function during tumor progression: regulation by distinct molecular mechanisms. J Immunol 2008; 180: 2011-7.

8. Nam SJ, Go H, Paik JH, et al. An increase of M2 macrophages predicts poor prognosis in patients with diffuse large B-cell lymphoma treated with rituximab, cyclophosphamide, doxorubicin, vincristine and prednisone. Leuk Lymphoma 2014; 55: 2466-76.

9. Kridel R, Steidl C, Gascoyne RD. Tumor-associated macrophages in diffuse large B-cell lymphoma. Haematologica 2015; 100: 143-5.

10. Marinaccio C, Ingravallo G, Gaudio F, et al. Microvascular density, CD68 and tryptase expression in human diffuse large B-cell lymphoma. Leuk Res 2014; 38: 1374-7.

11. Hoshino A, Costa-Silva B, Shen TL, et al. Tumour exosome integrins determine organotropic metastasis. Nature 2015; 527: 329-35.

12. Zhou W, Ke SQ, Huang Z, et al. Periostin secreted by glioblastoma stem cells recruits $M 2$ tumour-associated macrophages and promotes malignant growth. Nat Cell Biol 2015; 17: 170-82.

13. Ying X, Wu Q, Wu X, et al. Epithelial ovarian cancer-secreted exosomal miR-222-3p induces polarization of tumor-associated macrophages. Oncotarget 2016; 7 : 43076-87.

14. Sceneay J, Smyth MJ, Moller A. The pre-metastatic niche: finding common ground. Cancer Metastasis Rev 2013; 32: 449-64.

15. Bobrie A, Colombo M, Raposo G, Théry C. Exosome secretion: molecular mechanisms and roles in immune responses. Traffic 2011; 12: 1659-68.

16. Baig MS, Zaichick SV, Mao M, et al. NOS1-derived nitric oxide promotes NF-kappaB transcriptional activity through inhibition of suppressor of cytokine signaling-1. J Exp Med 2015; 212: 1725-38.

17. Yang Z, Xian $\mathrm{H}, \mathrm{Hu}$ J, et al. USP18 negatively regulates NF-kappaB signaling by targeting TAK1 and NEMO for deubiquitination through distinct mechanisms. Sci Rep 2015; 5: 12738

18. Mantovani A, Allavena P, Sozzani S, Vecchi A, Locati M, Sica A. Chemokines in the recruitment and shaping of the leukocyte infiltrate of tumors. Semin Cancer Biol 2004; 14: 155-60.

19. Condeelis J, Pollard JW. Macrophages: obligate partners for tumor cell migration, invasion, and metastasis. Cell 2006; 124: 263-6.

20. Ding P, Wang W, Wang J, Yang Z, Xue L. Expression of tumor-associated macrophage in progression of human glioma. Cell Biochem Biophys 2014; 70: 1625-31.

21. Mantovani A, Sozzani S, Locati M, Allavena P, Sica A. Macrophage polarization: tumor-associated macrophages as a paradigm for polarized M2 mononuclear phagocytes. Trends Immunol 2002; 23: 549-55.

22. Estko M, Baumgartner S, Urech K, et al. Tumour cell derived effects on monocyte/macrophage polarization and function and modulatory potential of Viscum album lipophilic extract in vitro. BMC Complement Altern Med 2015; 15: 130.

23. Hagemann T, Wilson J, Burke F, et al. Ovarian cancer cells polarize macrophages toward a tumor-associated phenotype. J Immunol 2006; 176: 5023-32.

24. Higginbotham JN, Demory Beckler M, Gephart JD, et al. Amphiregulin exosomes increase cancer cell invasion. Curr Biol 2011; 21: 779-86.

25. Ma H, Xu Y, Zhang R, Guo B, Zhang S, Zhang X. Differential expression study of circular RNAs in exosomes from serum and urine in patients with idiopathic membranous nephropathy. Arch Med Sci 2019; 15: 738-53.

26. Chow A, Zhou W, Liu L, et al. Macrophage immunomodulation by breast cancer-derived exosomes requires Toll-like receptor 2-mediated activation of NF-kB. Sci Rep 2014; 4: 5750.

27. Peinado H, Aleckovic M, Lavotshkin S, et al. Melanoma exosomes educate bone marrow progenitor cells toward a pro-metastatic phenotype through MET. Nat Med 2012; 18: 883-91.

28. Muller L, Mitsuhashi M, Simms P, et al. Tumor derived exosomes regulate expression of immune function-related genes in human T cell subsets. Sci Rep 2016; 6: 20254.

29. Théry C. Exosomes: secreted vesicles and intercellularcommunications. F1000 Biol Rep 2011; 3: 15.

30. Aung T, Chapuy B, Vogel D, et al. Exosomal evasion of humoral immunotherapy in aggressive B-cell lymphoma modulated by ATP-binding cassette transporter A3. Proc Natl Acad Sci USA 2011; 108: 15336-41. 
31. Movahedi K, Laoui D, Gysemans C, et al. Different tumor microenvironments contain functionally distinct subsets of macrophages derived from Ly6C(high) monocytes. Cancer Res 2010; 70: 5728-39.

32. Caras I, Tucureanu C, Lerescu L, et al. Influence of tumor cell culture supernatants on macrophage functional polarization: in vitro models of macrophage-tumor environment interaction. Tumori 2011; 97: 647-54.

33. Lu D, Peng F, Li J, et al. Urotensin II promotes secretion of LTB4 through 5-lipoxygenase via the UT-ROS-Akt pathway in RAW264.7 macrophages. Arch Med Sci 2019; 15: 1065-72.

34. Pollard JW. Tumour-educated macrophages promote tumour progression and metastasis. Nat Rev Cancer 2004; 4: 71-8. 